\title{
Modelling the Variable Angle Reflection and Transmission from Metamaterial Slabs
}

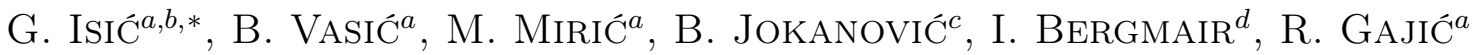 \\ AND K. HINGERL ${ }^{e}$ \\ ${ }^{a}$ Institute of Physics, Belgrade, Serbia \\ ${ }^{b}$ School of Electronic \& Electrical Engineering, University of Leeds, United Kingdom \\ ${ }^{c}$ IMTEL Communications A.D., Belgrade, Serbia \\ ${ }^{d}$ Profactor GmbH, Functional Surfaces and Nanostructures, Steyr-Gleink, Austria \\ e Zentrum für Oberflächen- und Nanoanalytik, Universität Linz, Austria
}

\begin{abstract}
We consider metamaterial slabs composed of perfectly conducting split-ring resonators. Assuming bianisotropic constitutive relations, we describe an $S$-parameter retrieval method that allows us to obtain the full material parameter tensors from numerically calculated $S$ parameters. The retrieval has been done for various angles of incidence, ranging from $0^{\circ}$ to $75^{\circ}$. It has been found that the parameters depend on the angle of incidence, but that this dependence is relatively small.
\end{abstract}

PACS numbers: 41.20.-q, 42.25.Bs, 42.70.Qs

\section{Introduction}

Electromagnetic (EM) metamaterials (MMs) are artificial structures made of unit cells (usually metallic) that exhibit a resonance at wavelengths large compared to the cell size. The long wavelength limit allows the description of MMs by effective constitutive parameters (e.g. $\varepsilon$ and $\mu$ ) while the resonant response yields values of $\varepsilon$ and $\mu$ not found in natural materials [1].

Two common methods are used to obtain the effective parameters of a MM: the $S$-parameter retrieval [2] and the field averaging method [3]. The first is based on measuring (or calculating) the $S$ parameters $\left(S_{11}\right.$, reflection and $S_{21}$, transmission) of a MM slab, assuming they correspond to a homogeneous slab and then using analytical inversion to find $\varepsilon$ and $\mu$ from $S_{11}$ and $S_{21}$. In the field averaging method, the $\boldsymbol{E}$ and $\boldsymbol{H}$ fields of propagating (Bloch) modes in an infinite MM are numerically calculated while effective $\varepsilon$ and $\mu$ are found by averaging the fields over a unit cell. In spectral regions where EM fields are not allowed to propagate (band gaps), one has to find the complex Bloch bands [4] to obtain $\varepsilon$ and $\mu$.

With the exception of [5-7], studies reported so far (numerical or experimental) assume the EM wave falling perpendicularly on the MM surface. Scalar $\varepsilon$ and $\mu$ obtained for perpendicular incidence cannot a priori be used for other angles because they do not account for

* corresponding author; e-mail: isicg@ipb.ac.rs (bi)anisotropy. Also, since MMs are operated in a regime where the wavelength is at most one order of magnitude larger than the unit cell, there is no a priori reason why the effective parameters would be angle-independent. Thus, investigating the consistency of effective parameters with respect to angle variation is of a fundamental significance in the design and application of MMs.

In this paper we describe a $S$-parameter retrieval method that yields the full constitutive tensors, $\bar{\varepsilon}, \bar{\mu}, \bar{\xi}$ and $\bar{\varsigma}$ of a bianisotropic MM slab. The tensorial nature of $\bar{\varepsilon}, \bar{\mu}, \bar{\xi}$ and $\bar{\varsigma}$ requires more than two $\mathrm{S}$ parameters to be known. Here we use all four $S$ parameters, $S_{11}$, $S_{12}, S_{21}=S_{12}$, [8], for two angles of incidence to recover four tensor elements that are assumed to describe the EM wave propagation in the effective medium.

\section{Constitutive relations and $S$-parameter retrieval}

Figure 1 shows a MM slab, (a) and (b), and the homogeneous bianisotropic slab, (c), assumed to be equivalent to the MM slab. Two unit cell orientations are considered as the MM slab shown in Fig. 1a effectively shows no bianisotropy, while the one in Fig. 1b is known to be bianisotropic [8-10].

The constitutive relations of a bianisotropic medium are [11]:

$$
D=\bar{\varepsilon} E+\bar{\xi} H, \quad B=\bar{\mu} H+\bar{\varsigma} E .
$$

For $\bar{\varepsilon}, \bar{\mu}, \bar{\xi}$, the following (implied by symmetry $[12,13]$ ) ansatz is introduced $[8,10]$ : 


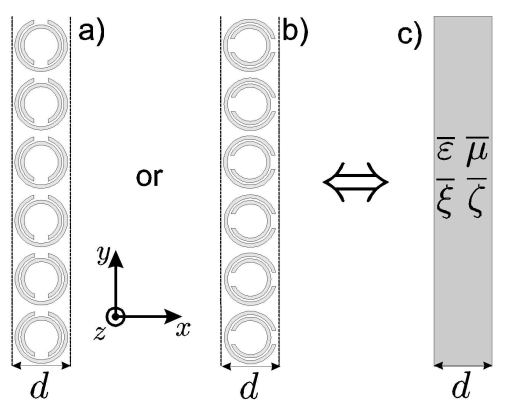

Fig. 1. (a) and (b) Two MM slab configurations considered in the paper. (c) The homogeneous (bi)anisotropic slab equivalent to the MM slab. In the $S$-parameter approach, this equivalence is assumed a priori.

$$
\begin{aligned}
& \bar{\varepsilon}=\left[\begin{array}{ccc}
\varepsilon_{x} & 0 & 0 \\
0 & \varepsilon_{y} & 0 \\
0 & 0 & \varepsilon_{z}
\end{array}\right], \quad \bar{\mu}=\left[\begin{array}{ccc}
\mu_{x} & 0 & 0 \\
0 & \mu_{y} & 0 \\
0 & 0 & \mu_{z}
\end{array}\right], \\
& \bar{\xi}=\left[\begin{array}{ccc}
0 & 0 & \xi_{x z} \\
0 & 0 & \xi_{y z} \\
\xi_{z x} & \xi_{z y} & 0
\end{array}\right],
\end{aligned}
$$

while $\bar{\varsigma}$ is found from $\bar{\xi}$ using the reciprocity criteria $[11,14], \bar{\varsigma}=-\xi^{\mathrm{T}}$, because the effective medium must be reciprocal if its constituents are reciprocal. For slabs perpendicular to the $x$ axis (see Fig. 1), $\xi_{x z}$ cannot be distinguished from $\mu_{z}$, i.e. this component of the magnetoelectric coupling tensor is redundant. We consider only the $\boldsymbol{H} \| z$ (TM) mode (the structure is 2D, i.e. invariant along the $z$-axis) in which case only $\varepsilon_{x}, \varepsilon_{y}, \mu_{z}, \xi_{y z}$ are relevant. The complementary tensor elements are found by considering the TE mode and the same retrieval method.

Figure 2 shows the simulation setup for COMSOL Multiphysics, the finite element method (FEM) solver we have used. The split-ring resonator is assumed to be made of a perfect electric conductor (PEC). The model is scalable so we have used an arbitrary unit of length denoted by $u$. Boundary conditions shown in Fig. 2 correspond to EM wave incident from the left, from which $S_{11}$ (reflection from left) and $S_{21}$ (transmission from left to right) are found. $S_{22}$ (reflection from right) and $S_{12}$ (transmission from right to left) are found by exchanging the position of the "incident field" and "zero field" boundary conditions so that the EM wave is incident from the right.

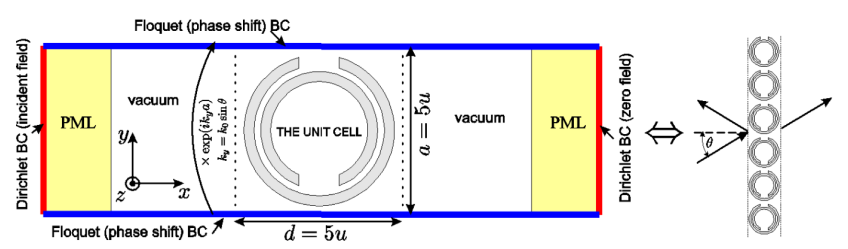

Fig. 2. Left: FEM model with boundary conditions and coordinate system orientation. The dimensions (along the $x$-direction) of the "vacuum" and "PML" (perfectly matched layer) domains are not in scale, in simulations much wider domains are used to ensure the decay of near fields (higher-order diffraction modes). Right: the physical meaning of the FEM model. Dashed lines denote the fictive limits of the equivalent homogeneous slab (as in Fig. 1) but have no physical meaning.

Consider a wave with vacuum wave vector $k_{0}$ propagating at angle $\theta$ with respect to the $x$-axis, having the $k_{x 1}=k_{0} \cos \theta$ and $k_{y}=k_{0} \sin \theta \boldsymbol{k}$-vector components. When refracted on the interface between vacuum and bianisotropic medium described by (2), the $x$-component of the $\boldsymbol{k}$-vector becomes

$$
k_{x 2}= \pm \sqrt{\varepsilon_{y}\left(\mu_{z}+\frac{\xi_{x z}^{2}}{\varepsilon_{x}}+\frac{\xi_{y z}^{2}}{\varepsilon_{y}}\right) k_{0}^{2}-\frac{\varepsilon_{y}}{\varepsilon_{x}} k_{y}^{2}} .
$$

The $S$ parameters of a slab of thickness $d$ are given by

$$
\begin{aligned}
& S_{21}=S_{12}=\frac{2 \mathrm{i} \varepsilon_{y} k_{x 1} k_{x 2}}{2 \mathrm{i} \varepsilon_{y} k_{x 1} k_{x 2} \cos \left(k_{x 2} d\right)+\left(\varepsilon_{y}^{2} k_{x 1}^{2}+k_{x 2}^{2}-\xi_{y z}^{2} k_{0}^{2}\right) \sin \left(k_{x 2} d\right)}, \\
& S_{11}=\frac{\left(\left(\varepsilon_{y} k_{x 1}+\xi_{y z} k_{0}\right)^{2}-k_{x 2}^{2}\right) \sin \left(k_{x 2} d\right)}{2 \mathrm{i} \varepsilon_{y} k_{x 1} k_{x 2} \cos \left(k_{x 2} d\right)+\left(\varepsilon_{y}^{2} k_{x 1}^{2}+k_{x 2}^{2}-\xi_{y z}^{2} k_{0}^{2}\right) \sin \left(k_{x 2} d\right)}, \\
& S_{22}=\frac{\left(\left(\varepsilon_{y} k_{x 1}-\xi_{y z} k_{0}\right)^{2}-k_{x 2}^{2}\right) \sin \left(k_{x 2} d\right)}{2 \mathrm{i} \varepsilon_{y} k_{x 1} k_{x 2} \cos \left(k_{x 2} d\right)+\left(\varepsilon_{y}^{2} k_{x 1}^{2}+k_{x 2}^{2}-\xi_{y z}^{2} k_{0}^{2}\right) \sin \left(k_{x 2} d\right)} .
\end{aligned}
$$

Defining $X, Y$ and $F$ as

$$
X=\frac{k_{x 2}}{\varepsilon_{y}}, \quad Y=k_{0} \frac{\xi_{y z}}{\varepsilon_{y}}, \quad F=\exp \left(\mathrm{i} k_{x 2} d\right),
$$

we can find them from $S$-parameters as

$$
X= \pm k_{x 1} \frac{\sqrt{\left(1+S_{21}^{2}-S_{11} S_{22}\right)^{2}-4 S_{21}^{2}}}{1+S_{11}-S_{21}^{2}+S_{22}+S_{11} S_{22}}
$$




$$
\begin{aligned}
& Y=k_{x 1} \frac{S_{11}-S_{22}}{1+S_{11}-S_{21}^{2}+S_{22}+S_{11} S_{22}}, \\
& F=\frac{1+S_{21}^{2}-S_{11} S_{22} \mp \sqrt{\left(1+S_{21}^{2}-S_{11} S_{22}\right)^{2}-4 S_{21}^{2}}}{2 S_{21}} .
\end{aligned}
$$

Now, using

$$
k_{x 2}=\frac{1}{d}(\arg \{F\}+2 m \pi-\mathrm{i} \ln |F|),
$$

and $(7), k_{x 2}, \varepsilon_{y}$ and $\xi_{y z}$ are found. $m$ is an integer that defines the branch of the complex logarithmic function. At low frequencies, such that the vacuum wavelength is large compared to the slab thickness $d, m$ is zero. The value of $m$ for higher frequencies is determined by the criteria that $k_{x 2}$ is a continuous function of $\omega$.

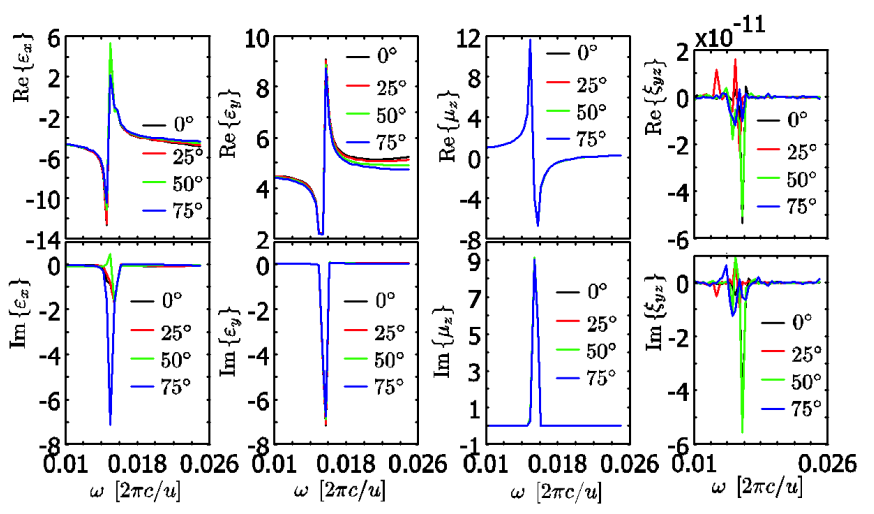

Fig. 3. Effective parameters retrieved for the slab shown in Fig. 1a. Values of $\xi_{y z}$ of the order $10^{-11}$ are much smaller than the accuracy of our solver, so we conclude that it is zero, as already found in [8] but for perpendicular incidence.

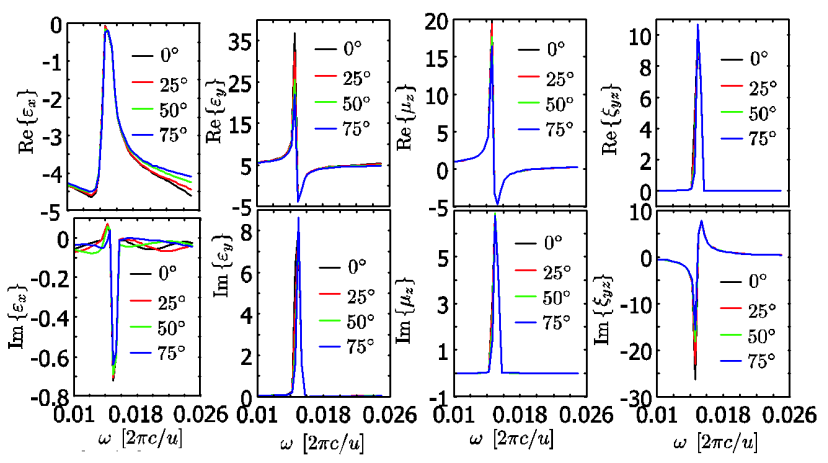

Fig. 4. Retrieved parameters for the slab shown in Fig. 1b. This slab is bianisotropic. The antiresonance in $\varepsilon_{x}$ (in this case) is a common feature of effective parameters found by $S$-parameter retrieval and has been reported by many other authors, see e.g. [15]. It does not imply that the retrieval procedure is erroneous, but rather that the effective parameters should be thought of as wave parameters rather than material parameters $[6,7]$.
The sign ambiguity in (3), (8) and (10) is resolved by the condition that the power flows from left to right. This means that the imaginary part of $k_{x 2}$ is positive, the real part of $X$ is positive and $|F|$ is smaller (or equal in a lossless MM) than 1 . In practice, a very small loss is introduced in the MM unit cell to avoid errors due to finite numerical accuracy. If we repeat the same procedure but for a slightly different angle $(\Delta \theta=\pi / 180$ has been used), we obtain $\mu_{z}$ and $\varepsilon_{x}$ from (3).

Using the above described method, we have calculated $\varepsilon_{x}, \varepsilon_{y}, \mu_{z}, \xi_{y z}$, for the two slabs shown in Fig. 1a and b at four different angles of incidence. These are displayed in Figs. 3 and 4.

It has been found that the retrieved parameters depend on the angle of incidence. However, the degree of such a dependence is relatively small in the investigated case, so that the effective tensors $\bar{\varepsilon}, \bar{\mu}, \bar{\xi}$ and $\bar{\varsigma}$ may still offer a useful description of the MM slab.

\section{Conclusion}

A numerical method to retrieve the full bianisotropic constitutive tensors of a 2D MM slab has been described and applied to retrieve the parameters of two slabs made of PEC split-ring resonators. The method relies on calculating the reflection, $S_{11}$ and $S_{22}$, and transmission, $S_{12}$ and $S_{21}$, coefficients (from both sides of the slab) for two different angles and obtaining the material parameters from analytical formulae that apply to a homogeneous slab.

The effective parameters have been found to depend on the angle of incidence implying that an effective medium with spatial dispersion would describe the MM slab more accurately. However, the effective parameters of the particular MM slab investigated here show only a weak angle-dependence around the resonant frequency, so they might still be a useful concept in understanding the EM wave propagation through the MM.

\section{Acknowledgments}

This work is supported by the Serbian Ministry of Science under project No. 141047. G.I. acknowledges support from ORSAS in the U.K. and the University of Leeds. R.G. acknowledges support from EU FP7 projects Nanocharm and NIMNIL. K.H. is grateful to the Austrian NIL-meta-NIL Austria project from FFG for partial support. We thank Johann Messner from the Linz Supercomputer Center for technical support. 


\section{References}

[1] S.A. Ramakrishna, T.M. Grzegorczyk, Physics and Applications of Negative Refractive Index Materials, CRC Press, New York 2009.

[2] X. Chen, T.M. Grzegorczyk, B.-I. Wu, J. Pacheco, Jr., J.A. Kong, Phys. Rev. E 70, 016608 (2004).

[3] D.R. Smith, J.B. Pendry, J. Opt. Soc. Am. B 23, 391 (2006).

[4] M. Davanco, Y. Urzhumov, G. Shvets, Opt. Express 15, 9681 (2007).

[5] T. Driscoll, D.N. Basov, W.J. Padilla, J.J. Mock, D.R. Smith, Phys. Rev. B 75, 115114 (2007).

[6] C. Menzel, C. Rockstuhl, T. Paul, F. Lederer, Phys. Rev. B 77, 195328 (2008).

[7] C. Menzel, T. Paul, C. Rockstuhl, F. Lederer, T. Pertsch, S. Tretyakov, arXiv:0908.2393v1 [physics.optics] (2009).
[8] Z. Li, K. Aydin, E. Ozbay, Phys. Rev. E 79, 026610 (2009).

[9] R. Marques, F. Medina, R. Rafii-El-Idrissi, Phys. Rev. B 65, 144440 (2002)

[10] X. Chen, B.-I. Wu, J.A. Kong, T.M. Grzegorczyk, Phys. Rev. E 71, 046610 (2005).

[11] J.A. Kong, Electromagnetic Wave Theory, Wiley, New York 1986

[12] W.J. Padilla, Opt. Express 15, 1639 (2007).

[13] O. Isik, K.P. Esselle, Metamaterials 3, 33 (2009).

[14] C.M. Krowne, IEEE Trans. Antennas Propagat. AP-32, 1224 (1984).

[15] Th. Koschny, P. Markoš, E.N. Economou, D.R. Smith, D.C. Vier, C.M. Soukoulis, Phys Rev. B 71, 245105 (2005) 